\title{
Identification and Validation of Stable Reference Genes for Gene Expression Analysis in Sword-leaf Dogbane Using Quantitative Reverse Transcriptase Polymerase Chain Reaction
}

\author{
Zongchang $\mathrm{Xu}^{1}$ \\ Marine Agriculture Research Center, Tobacco Research Institute, Chinese Academy of Agricultural \\ Sciences, Qingdao, 266101, China \\ Meng Wang ${ }^{1}$ \\ Key Laboratory for Tobacco Gene Resources, Tobacco Research Institute, Chinese Academy of \\ Agricultural Sciences, Qingdao, 266101, China \\ Jinhui Zhou \\ Medical College, Pharmic Department, Qingdao University, Qingdao 266021, China \\ Han Liu \\ College of Agriculture, Qingdao Agricultural University, Qingdao, 266109, China
}

Chengsheng Zhang ${ }^{2}$ and Yiqiang $\mathbf{L i}^{2}$

Marine Agriculture Research Center, Tobacco Research Institute, Chinese Academy of Agricultural Sciences, Qingdao, 266101, China

\begin{abstract}
AdDitional INDEX words. Apocynum venetum, flavonoid, luobuma, normalization, salt stress
Abstract. Sword-leaf dogbane (Apocynum venetum) is a traditional Chinese herb with increasingly recognized potential to enhance health, but no study of stable reference genes in this herb has been reported. Based on a homologous cloning strategy, we have successfully cloned five candidate reference genes from sword-leaf dogbane: glyceraldehyde-3-phosphate dehydrogenase $(A v G A P D H)$, beta tubulin $(A v b T U B)$, polyubiquitin $(A v U B Q)$, elongation factor 1-alpha $(A v E F 1 \alpha)$, and actin $(A v A C T I N)$. Three distinct algorithms, geNorm, NormFinder, and BestKeeper, were used to estimate the expression stability of candidate reference primer pairs. We found that $A v A C T I N-2$ and $A v A C T I N-3$ presented the highest stability of expression in different tissue samples, and $A v G A P D H-2$ was most stable under salinity stress. In addition, we illustrated the application of these new reference genes by assaying the expression levels of two hyperoside biosynthesis terminal enzyme genes, flavonoid $3^{\prime}$-hydroxylase $\left(F 3^{\prime} H\right)$ and flavonol synthase $(F L S)$, under salinity stress. Our study is the first to report stable expression of internal reference genes in sword-leaf dogbane in multiple experimental sample sets.
\end{abstract}

Quantitative reverse transcriptase polymerase chain reaction (qRT-PCR) is now widely used in molecular experiments in diverse fields, including molecular medicine, biotechnology, microbiology, and human clinical diagnostics (Bustin and Dorudi, 1998; Bustin and Mueller, 2005; Nolan et al., 2006). It is especially widely used in plant molecular research (Gachon et al., 2004). Compared with other time-consuming experimental techniques used to quantify the abundance of RNA transcripts, such as northern blotting (Artico et al., 2010), qRT-PCR is more rapid and more sensitive, being capable of detecting gene expression at a lower level (Nolan et al., 2006; Wong and Medrano, 2005). Although qRT-PCR is a reliable and efficient technique to evaluate variation in gene expression level, diverse factors can affect the accuracy of its results. These include variability of RNA templates or of cDNA synthesis efficiency in different reactions, inappropriate data normalization methods, and variation in materials caused by stressors (Liu

Received for publication 25 Sept. 2018. Accepted for publication 24 Oct. 2018. ${ }^{1}$ These authors contributed equally.

2Corresponding authors. E-mail: zhangchengsheng@caas.cn or liyiqiang@caas.cn. et al., 2014; Nolan et al., 2006). However, these issues can be offset by the use of an optimal reference gene that exhibits stable expression levels in diverse experimental conditions or tissues (Huggett et al., 2005; Radoni et al., 2003). Therefore, identification of stable reference genes provides an important foundation for molecular research.

Housekeeping genes, such as $A C T I N, G A P D H, E F 1 \alpha, U B Q$, $b$-TUB, heat shock protein (HSP), $18 \mathrm{~S}$ ribosomal RNA (18SrRNA), and $28 \mathrm{~S}$ ribosomal RNA (28SrRNA), have been applied as reference genes for expression data normalization in many plant species (Aithal and Rajeswari, 2015; Dean et al., 2002; Garg et al., 2010; Jain et al., 2006; Sinha et al., 2015; Sturzenbaum and Kille, 2001). However, reported reference genes can exhibit inconstant expression levels under different experimental conditions (Hruz et al., 2011; Maroufi et al., 2010). For example, the expression of common reference genes in Arabidopsis thaliana, including AtACTIN, AtTUB, AtUBQ, and $A t E F$, was reported to be highly variable during development (Gutierrez et al., 2008a). The instability in expression level of these reference genes implies that complex considerations are relevant to their selection (Gutierrez et al., 2008b). 
For example, reference genes that are effective in one species are not necessarily useful in another species (Liu et al., 2014). Hence, screening and validation of internal reference genes under a variety of experimental conditions is relevant and important work in any species subject to molecular research.

Sword-leaf dogbane, called "luobuma" in China, is an important traditional medicinal herb. Leaf extracts from sword-leaf dogbane contain many potentially useful chemical components, including flavonoids, triterpenoids, organic acids, and amino acids (Xie et al., 2012). As outlined in our recent review, sword-leaf dogbane research now mainly focuses on the chemical components and medicinal effects of extracts $(\mathrm{Xu}$ et al., 2018). The flavonoids extracted from sword-leaf dogbane leaves have proven medicinal effects against multiple diseases, including hypertension, atherosclerosis, and hyperlipidemia (Kim et al., 2000; Lv et al., 2016; Wang et al., 2015). Furthermore, sword-leaf dogbane leaf tea has become increasingly popular in both North American and East Asian health food markets (Xie et al., 2012). Without doubt, sword-leaf dogbane has great potential application in medicine and health. However, molecular research data on sword-leaf dogbane are seldom reported. The rapid, large-scale genome sequencing of various medicinal herbs in recent years suggests that the growth of research on medicinal herbs at a molecular level is becoming a trend. The availability of transcriptomic, metabolomic, and genomic techniques therefore presents a timely opportunity to promote the knowledge and application of sword-leaf dogbane. Any prospective investigation into the expression levels of the genes that encode the biosynthetic enzymes of its key medicinal components or regulate the progress of biosynthesis will benefit from fundamental molecular research. Thus, identification of stably expressed internal reference genes in sword-leaf dogbane is particularly relevant now.

In this study, five housekeeping genes were cloned to identify the most stable internal reference genes for qRT-PCR data normalization. A total of six pairs of $\mathrm{qRT}-\mathrm{PCR}$ primers, covering all of the five candidate reference genes, were validated in different tissues and salinity stress conditions. To evaluate the usefulness of new internal reference genes, the expression profiles of two functional genes, $F 3^{\prime} H$ and FLS, which encode two hyperoside biosynthesis terminal enzymes, were analyzed following salinity stress.

\section{Materials and Methods}

Plant material and salinity stress treatments. Apocynum venetum possesses developed perennial roots and is propagated through seeds and perennial roots (Zhang et al., 2005). In the wild, sword-leaf dogbane can quickly form dominant communities through perennial roots propagation (Xu et al., 2018; Zhang et al., 2005). Therefore, in the present study, we considered a sword-leaf dogbane population grown within a 5-m-diameter circle to have similar genetic background. Sword-leaf dogbane was obtained from Dongying City, Shandong Province of China. The perennial roots of adult plants that had lived more than 3 years in the wild were transferred to field plots defined by a circle $5 \mathrm{~m}$ in diameter to pots at the end of Mar. 2018, and seeds were collected from the same place in Sept. 2017. Seeds were washed three times with double-distilled water and germinated on wet filter paper covered by a culture dish at room temperature. Germinated seeds were then transferred to a plastic pot. Seedlings and adult plants were grown in a greenhouse at $26 \pm 1{ }^{\circ} \mathrm{C}$ with $16 / 8-\mathrm{h}$ light/dark photoperiod cycles. The young leaves, stems, and roots were dissected from at least 10 seedlings, and senescent leaves, flowers, and perennial roots were obtained from five adult plants. The same tissues were mixed and immediately frozen in liquid nitrogen, and stored at $-80{ }^{\circ} \mathrm{C}$ for further study.

For salinity stress treatments, 200, 400, 600, and $800 \mathrm{~mm}$ $\mathrm{NaCl}$ solutions were added to the pots containing adult plants. Mature leaves were collected from untreated (control) plants and treated adult plants after 3 and $7 \mathrm{~d}$. Samples were flashfrozen in liquid nitrogen immediately and at $-80{ }^{\circ} \mathrm{C}$ for further analyses.

Total RNA EXtraction AND CDNA sYNThesis. Total RNA of all samples was extracted using a MiniBEST Plant RNA Extraction Kit (code no. 9769; TaKaRa, Dalian, China) according to the manufacturer's instructions. As part of the extraction protocol, DNaseI was used to digest genomic DNA contamination. The integrity of RNA was assessed by $1.2 \%$ agarose gel electrophoresis. Two intact and clear RNA bands (28S and 18S) indicated successful extraction. The quality and quantity of total RNA were measured by a spectrophotometer (NanoDrop 2000 ${ }^{\circledR}$; Thermo Fisher Scientific, Waltham, MA). Samples showing a 260/280 ratio between 1.8 and 2.0, and a 260:230 ratio between 2.0 and 2.4, were used for further analysis. A total of $1 \mu \mathrm{g}$ of purified RNA from each sample was used to synthesize first-strand cDNA according to the manufacturer's instructions [HiScript ${ }^{\circledR} \mathrm{Q}$ RT SuperMix for qPCR (+gDNA wiper) Kit; Vazyme, Nanjing, China]. Finally, $20 \mu \mathrm{L}$ cDNA products were diluted into $200 \mu \mathrm{L}$ for qRT-PCR. A mixture of an equal volume from a cDNA pool of all samples was used as the template to amplify the candidate sword-leaf dogbane reference genes to test the specificity and efficiency of our qRT-PCR primer pairs.

Candidate Reference Gene Fragment homologous AMPLIFICATION. Because of the lack of knowledge of the sword-leaf dogbane genome, we first employed a homologous cloning strategy to obtain the sequences of sword-leaf dogbane candidate reference genes. A set of previously published reference genes including $E F 1 \alpha, G A P D H, A C T I N 1, U B Q 10$, and $b-T U B$ from $A$. thaliana or Oryza sativa (Garg et al., 2010; Jain et al., 2006; Sinha et al., 2015) was initially selected for testing in sword-leaf dogbane. Coding sequences of $A$. thaliana and $O$. sativa reference genes ACTIN1 (AT2G37620), GAPDH1 (AT1G16300), b-TUB (AT1G75780), UBQ10 (AK101547), and $E F 1 \alpha(A J 004960)$ were downloaded from the GenBank database as queries for a basic local alignment search tool (BLAST) search to obtain the sequences of homologous genes from other species. For each of the candidate genes, homologous sequences from at least 10 other species were collected. Multiple sequence alignments of these homologues were performed using the ClustalW function of the MEGA 6.0 software (Wang et al., 2018). The highly homologous segments were used to design universal primers containing several annexation bases to amplify sword-leaf dogbane homologous genes. The lengths of amplification fragments ranged from 500 to $800 \mathrm{bp}$. Universal primers are listed in Supplemental Table 1. Positive PCR products were Sanger sequenced to obtain partial sequences. After Sanger sequencing, candidate reference genes of swordleaf dogbane were identified by sequence comparison against the corresponding genes of $A$. thaliana or $O$. sativa. Furthermore, the gene type of sequenced genes was also identified 
using the National Center for Biotechnology Information (NCBI) BLAST tool. To extend the sequence of five sword-leaf dogbane candidate reference genes, a 3 ' rapidamplification of cDNA ends (RACE)-like primer, Xu-1117, GACTCGAGTCGACATCGATTTTTTTTTTTTTTTTT, was used instead of Oligo dT for the first cDNA strand synthesis. The cDNA was used as a template to extend the $3^{\prime}$ terminal sequence using the universal forward primers of each swordleaf dogbane candidate reference gene and $\mathrm{Xu}-1118$, GACTCGAGTCGACATCGATTTT. Finally, the partial gene sequence was used to design qRT-PCR primers.

Primer design and Selection. The OligoCalc tool (Kibbe, 2007) was used to design qRT-PCR primers. To assess the validity of the potential reference genes as thoroughly as possible, at least two primer pairs were designed for each candidate gene, with one set close to the stop codon position and another far from that position. The annealing temperature of all the primers was $\approx 58^{\circ} \mathrm{C}$, and their length ranged from 21 to 23 nucleotides. Before qRT-PCR, the RT-PCR products of all primer pairs were electrophoresed on $3 \%$ agarose gel and stained by ethidium bromide to verify successful amplification and the specificity of the primer pairs. The fragments were then cloned into pEASY-T1 Cloning Vector (CT101-01; TransGen, Beijing, China) to and sequenced to confirm amplification of the correct products. In addition, the specificity of the primer pairs was also validated by melting-curve analyses following amplification by qRT-PCR.

AMPLIFICATION EFFICIENCY TEST OF QRT-PCR PRIMER PAIRS. We performed qRT-PCR analysis on an Applied Biosystems 7500 Real-Time PCR system (Thermo Fisher Scientific) according to the manufacturer's instructions. The volume of qRT-PCR reactions was $20 \mu \mathrm{L}$, including $1 \mu \mathrm{L}$ cDNA, $10 \mu \mathrm{L}$
$2 \times$ SYBR green mix (Q321, Vazyme), $0.4 \mu \mathrm{L}$ ROX Reference Dye, and $0.4 \mu \mathrm{L}$ of each forward and reverse primer $(10 \mu \mathrm{M})$. Reaction mixtures were filled up to $20 \mu \mathrm{L}$ with water. The PCR conditions were as follows: $50^{\circ} \mathrm{C}$ for $2 \mathrm{~min}$ and $95^{\circ} \mathrm{C}$ for $5 \mathrm{~min}$, followed by 40 cycles of $95{ }^{\circ} \mathrm{C}$ for $10 \mathrm{~s}$ and $60{ }^{\circ} \mathrm{C}$ for $35 \mathrm{~s}$. The melting curves were analyzed at 95 to $60{ }^{\circ} \mathrm{C}$ after 40 cycles. Each qRT-PCR was performed in triplicate. To evaluate the amplification efficiency of all primer pairs, cDNA mixture pools were serial-diluted 10-fold to use as templates for qRTPCR. Cycle threshold $(\mathrm{Ct})$ values were used to calculate amplification efficiency and correlation coefficient. The amplification efficiency (E) of each primer pair was calculated according to the following equation: $\mathrm{E}=10^{-1 / \text { slope }}-1$ (Kong et al., 2014), where the slope is derived from the regression equation, calculated using Excel (Microsoft Corp., Redmond, WA).

AsSESSMent of STABLE REFERENCE PRIMER PAIRs. We used three statistical algorithms to evaluate the stability of the candidate reference gene primer pairs: geNorm (Vandesompele et al., 2002), BestKeeper (Pfaffl et al., 2004), and NormFinder (Andersen et al., 2004). The parameters calculated by geNorm were the average gene expression stability measure (M) and the average pairwise variation (V). The recommended upper threshold of the $M$ value is 1.5. Smaller $M$ values indicate more stable expression. Conversely, a gene with a high $\mathrm{M}$ value is less stable (Hong et al., 2008; Vandesompele et al., 2002). The pairwise variation $\mathrm{V}_{\mathrm{n}} / \mathrm{V}_{\mathrm{n}+1}$ represents the influence of increasing the expression of a reference gene on the normalized expression results. $A V_{n} / V_{n+1}$ threshold below 0.15 indicates that the variation in the reference gene will not significantly affect the final results and additional reference genes are not required (Liu et al., 2014; Vandesompele et al., 2002). The

Table 1. Summary of amplification characteristics of candidate reference gene primer pairs of sword-leaf dogbane used for quantitative reverse transcriptase polymerase chain reaction analysis.

\begin{tabular}{|c|c|c|c|c|c|c|}
\hline Gene & Primer pair & Sequence $\left(5^{\prime}-3^{\prime}\right)$ & Position & Length (bp) & $\mathrm{E}(\%)^{\mathrm{z}}$ & $R^{2}$ \\
\hline \multirow[t]{4}{*}{$\overline{A v A C T I N}$} & $A v A C T I N-1 F$ & GCTAATAGAGAGAAGATGACTC & $5^{\prime}$ & 91 & 76.84 & 0.9905 \\
\hline & $A v A C T I N-2 F$ & CAGCATGAAGATTAAGGTGGTT & $3^{\prime}$ & 122 & 93.98 & 0.9997 \\
\hline & $A v A C T I N-3 F$ & TACATGTTCACCACCTCTGCT & Middle & 120 & 92.82 & 0.9988 \\
\hline & $A v A C T I N-3 R$ & CTCAAGAGAAGAGCTGCTTTTTG & & & & \\
\hline \multirow[t]{2}{*}{$A v G A P D H$} & $A v G A P D H-1 F$ & TGGTGGGAGTAAATGAGTTTAC & $5^{\prime}$ & 116 & 85.01 & 0.9957 \\
\hline & $A v G A P D H-2 R$ & ATCGTCATCAGTGTATCCAAGA & & & & \\
\hline \multirow[t]{4}{*}{$A v b T U B$} & $A v b T U B-1 F$ & TCACTTGATATCTGCTACGATG & Middle & 97 & 85.95 & 0.9885 \\
\hline & $A v b T U B-1 R$ & GATAAGGTTGACAGCAAGCTTC & & & & \\
\hline & $A v b T U B-2 F$ & CTGAGAATTGTGACTGCCTTCA & $5^{\prime}$ & 121 & 91.05 & 0.9969 \\
\hline & $A v b T U B-2 R$ & CTGAGAATTGTGACTGCCTTCA & & & & \\
\hline & $A v U B Q-2 F$ & TAACGTGAAGGCTAAAATCCAG & $3^{\prime}$ & 119 & 94.22 & 0.984 \\
\hline & $A v U B Q-2 R$ & СCTTCTGAATGTTGTAGTCAGC & & & & \\
\hline \multirow[t]{4}{*}{$A v E F 1 \alpha$} & $A v E F 1 \alpha-1 F$ & TGAGAGGTCTACCAACCTTGA & $5^{\prime}$ & 75 & 98.30 & 0.9902 \\
\hline & $A v E F 1 \alpha-1 R$ & TTGGGCTCAGAAATCAGGTCAA & & & & \\
\hline & $A v E F 1 \alpha-2 F$ & ATATGCTCCAGTGCTTGACTG & $3^{\prime}$ & 99 & 74.82 & 0.9976 \\
\hline & $A v E F 1 \alpha-2 R$ & TTCTCGAGCTCCTTACCAGAT & & & & \\
\hline
\end{tabular}

${ }^{\mathrm{z}}$ Amplification efficiency of primer pairs. 
parameter calculated by NormFinder was the stability value that is related to the systematic error of each candidate gene. Smaller values indicate more stable gene expression (Andersen et al., 2004). The parameter calculated using BestKeeper was the coefficient of correlation $(r)$ value. The closer this value is to one, the more stable is the gene's expression (Pfaffl et al., 2004).
To further characterize the candidate reference primer pairs under specific experimental conditions, 18 samples were divided into two subsets. To test the primers on different tissues, we used eight samples including adult perennial roots, seedlings, germination seeds, phloem, adult young leaf, adult senescent leaf, seedling leaf, and flower. Our salinity-stressed sample set comprised 10 leaf samples collected from the adult

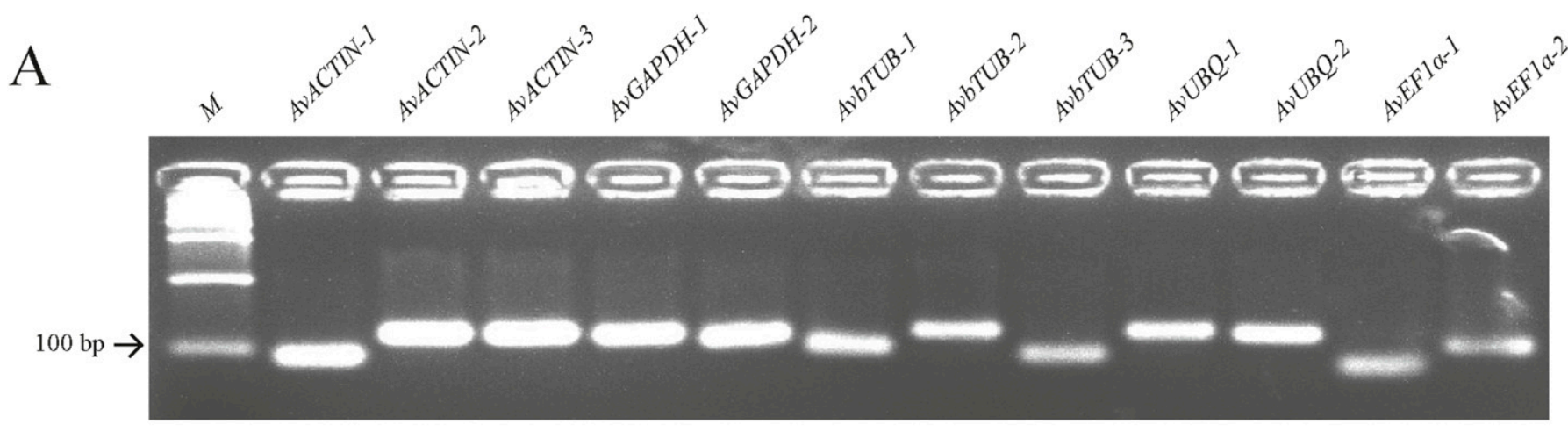

B

AvACTIN-1

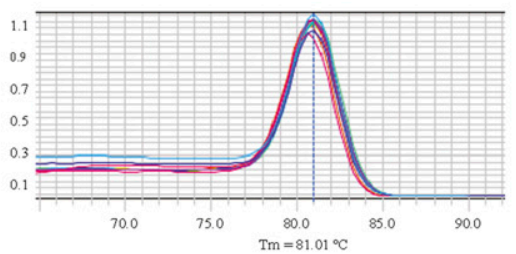

AvGAPDH-1

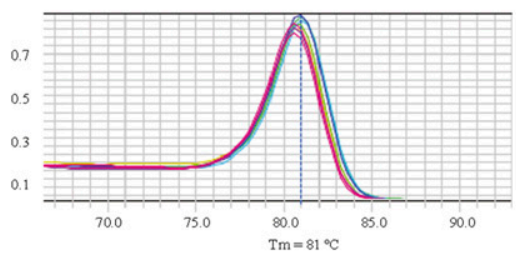

$A v b T U B-2$

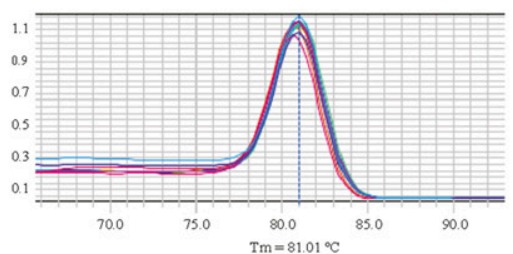

$A v U B Q-2$

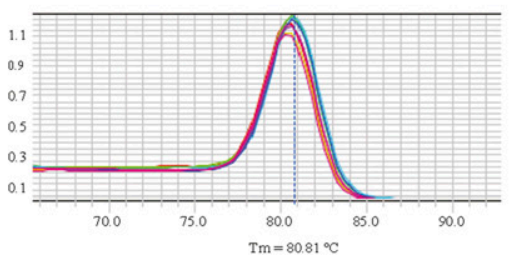

AvACTIN-2

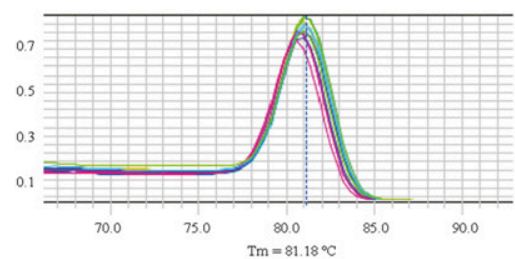

$A v G A P D H-2$

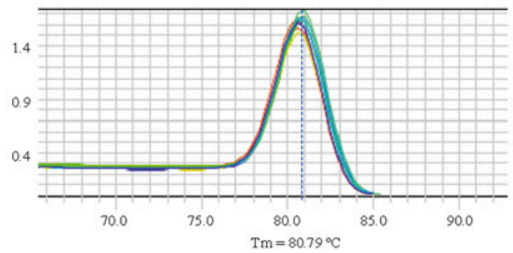

AvbTUB-3

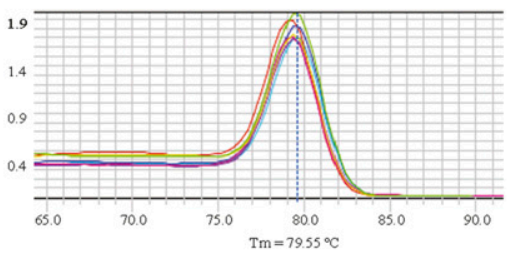

$A v E F 1 \alpha-1$

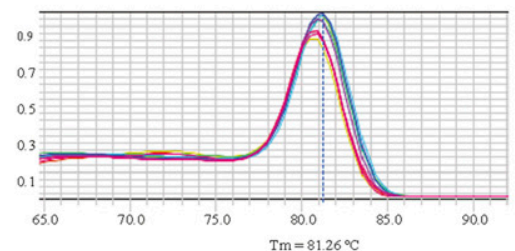

$A v A C T I N-3$

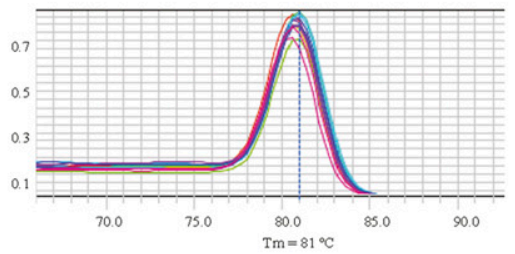

$A v b T U B-1$

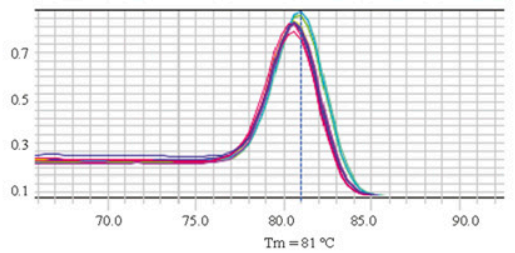

$A v U B Q-1$

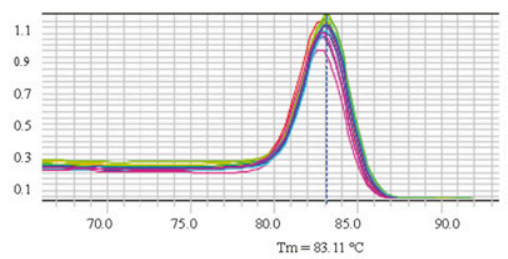

$A v E F 1 \alpha-2$

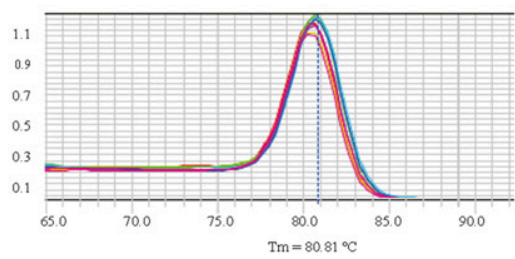

Fig. 1. Polymerase chain reaction amplification specificity and melting curves $(\mathrm{Tm}=$ melting temperature $)$ of 12 pairs of primers of sword-leaf dogbane. $(\mathbf{A})$ Only a single amplification product for each primer pair was identified by $3 \%$ agarose gel electrophoresis. $A v A C T I N=$ sword-leaf dogbane actin gene; $A v b T U B=$ swordleaf dogbane beta tubulin gene; $A v E F 1 \alpha=$ sword-leaf dogbane elongation factor 1-alpha gene; $A v G A P D H=$ sword-leaf dogbane glyceraldehyde-3-phosphate dehydrogenase gene; $A v U B Q=$ sword-leaf dogbane polyubiquitin gene; $\mathrm{M}=$ Marker 1000 Plus. (B) Melting curves of 12 candidate reference gene primer pairs. 
plants, to which were added $200,400,600$, or $800 \mathrm{~mm} \mathrm{NaCl}$ solution after 3 and $7 \mathrm{~d}$, respectively. Adult plant leaves given no special treatment were used as the control.

\section{Results}

Homologous CLONING OF SWORD-LEAF DOGBANE CANDIDATE REFERENCE GENES. Given that no large-scale transcriptome or genome sequences were available in public databases, we used a homologous cloning strategy to obtain the sequences of sword-leaf dogbane candidate reference genes. Positive products of $A v G A P D H, A v U B Q, A v E F 1 \alpha, A v A C T I N$, and $A v b T U B$ were obtained. After Sanger sequencing and BLAST against the NCBI database, the length of these five candidate reference genes was extended to nearly $1 \mathrm{~kb}$ by a $3^{\prime}$ RACE amplification (Supplemental Table 2). These partial sequences were used to design qRT-PCR primers.

QRT-PCR PRIMER DESIGN, SPECIFICITY, AND AMPLIFICATION EFFICIENCY TEST. To assess the validity of the potential reference genes as far as possible, 12 primer pairs were designed based on the sequences of five candidate reference genes (Table 1). Two pairs of primers were designed for

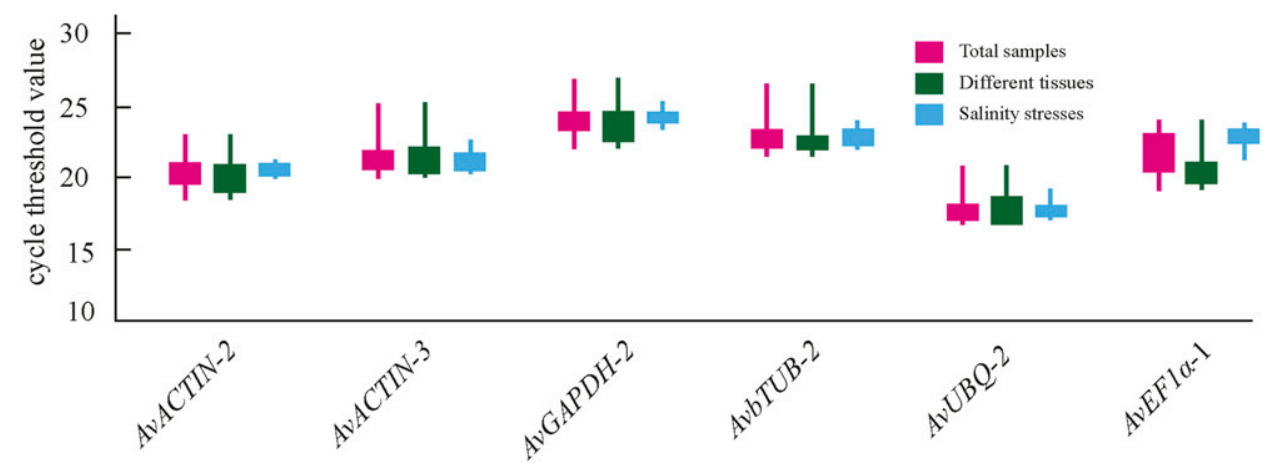

Fig. 2. Boxplots exhibiting the expression profiling of six candidate reference primer pairs of sword-leaf dogbane represented by raw cycle threshold values. The data were represented by raw cycle threshold values in different tissues sample set, salinity stresses sample set, and total samples set. Boxes exhibit the 25 th/75th percentiles, the line marks the median, squares represent the means, and whiskers indicate the ranges for the total samples.

A

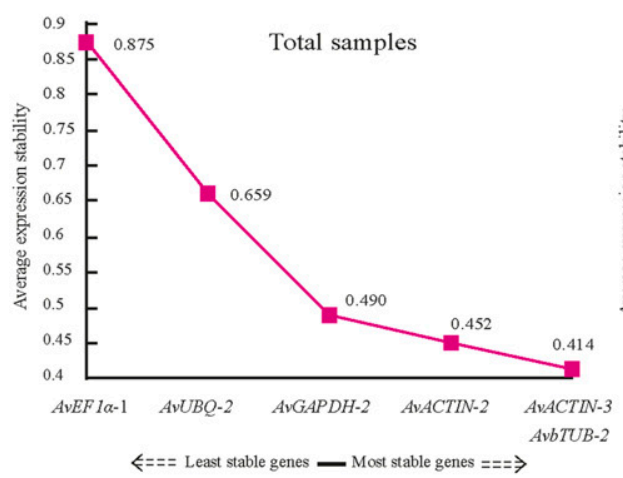

$\mathrm{D}$

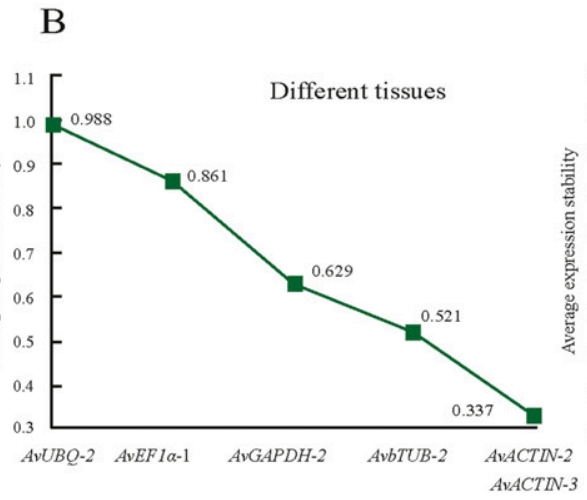

$\Leftrightarrow===$ Least stable genes - Most stable genes $===\Rightarrow$
$\mathrm{C}$

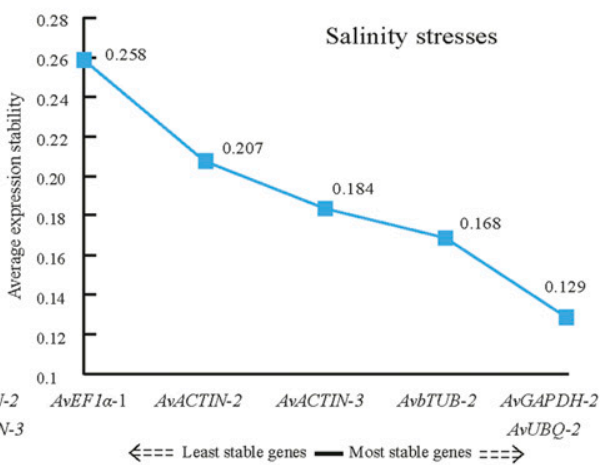

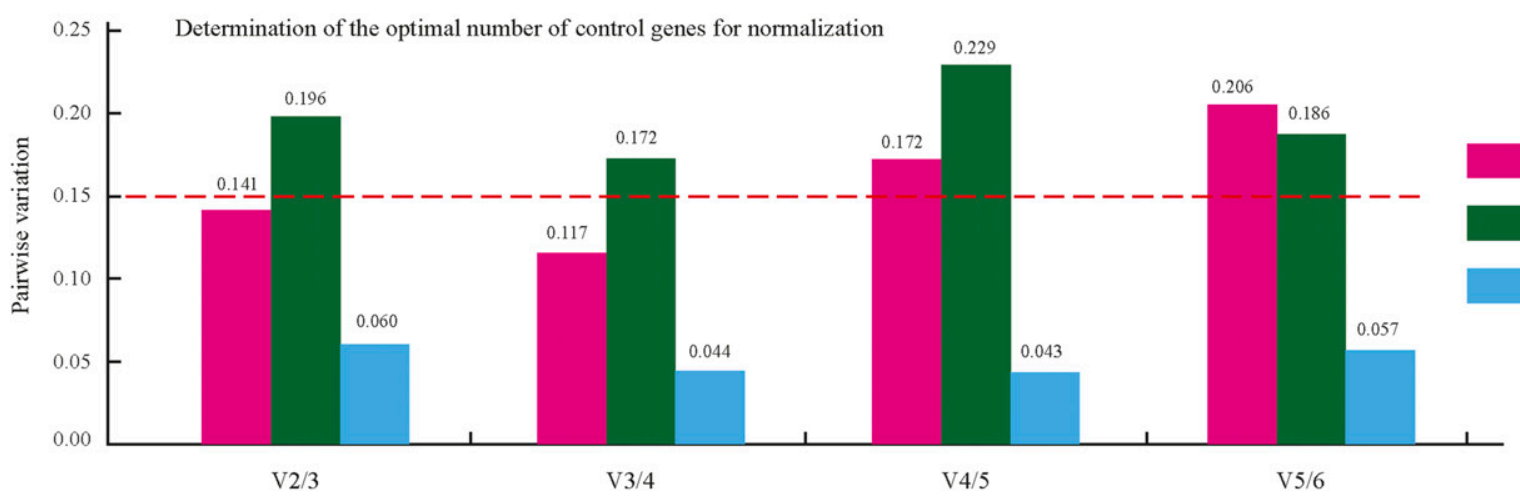

Fig. 3. Gene expression stability values $(\mathrm{M})$ and pairwise variation $(\mathrm{V})$ of candidate primer pairs of sword-leaf dogbane validated by geNorm program (Vandesompele et al., 2002). The M values were calculated based on the average pairwise variation existing between all pairs of the candidate genes for samples in (A) total samples set, (B) different tissues samples set, and (C) salinity stress samples set. A lower M value indicates more stable expression. (D) The optimal number of reference genes required for effective normalization. The pairwise variation $\left(\mathrm{V}_{\mathrm{n}} / \mathrm{V}_{\mathrm{n}+1}\right)$ was analyzed between the normalization factors NFn and $\mathrm{NFn}+1$. When $\mathrm{V}_{\mathrm{n}} / \mathrm{V}_{\mathrm{n}+1}$ is less than 0.15 , then the optimal number of reference genes is $\mathrm{N}$. 
$A v G A P D H, A v U B Q$, and $A v E F 1 \alpha$, and three pairs of primers were designed for $A v A C T I N$ and $A v b T U B$. Amplification specificity for each pair of primers was checked by electrophoresis in $3 \%$ agarose gel using cDNA as a template. All the primer pairs yielded a clear, strong, and single band with no primer-dimer formation, indicating the specificity of the primer pairs (Fig. 1A). Subsequently, melting curve analyses were conducted for all the primer pairs. The presence of a single peak, and no visible primer-dimer formation, further confirmed the efficiency of each primer pair. The melting temperatures (Tm) of all PCR products ranged from $79.55{ }^{\circ} \mathrm{C}$ for $A v b T U B-3$ to $83.1{ }^{\circ} \mathrm{C}$ for $A v U B Q-1$ (Fig. 1B). The qRT-PCR efficiency of all the 12 primer pairs ranged from $65.19 \%(A v b T U B-3)$ to $98.30 \%(A v E F 1 \alpha-1)$. The determination coefficients $\left(R^{2}\right)$ of the regression equation varied from 0.984 for $A v U B Q-2$ to 0.9997 for $A v A C T I N-2$ (Table 1).

EXPRESSION PROFILING OF CANDIDATE REFERENCE GENES IN DIFFERENT TISSUES AND AT DIFFERENT SALINITY STRESSES. For expression profiling analyses, only primer pairs that showed amplification efficiency between $90 \%$ and $100 \%$ were used (Pfaffl et al., 2004). In total, six pairs of primers (Table 1) were used to amplify the 18 samples collected from different swordleaf dogbane tissues (normal growth conditions, and leaves stressed with different $\mathrm{NaCl}$ concentrations), by qRT-PCR. The results are presented as $\mathrm{Ct}$ values. These values varied widely among the six pairs of primers, ranging from 16.2 to 26.2 cycles, but most of the $\mathrm{Ct}$ values were between 19.7 and 23.4 cycles (Fig. 2). AvGAPDH-2, which had the highest average mean $\mathrm{Ct}$ value of 23.4, was expressed at the lowest level among the six candidate reference gene primer pairs. By contrast,
$A v U B Q-2$, with the lowest average $\mathrm{Ct}$ value of 17.2 , exhibited the highest expression level. We observed 3.8 to 5.1 cycles of $\mathrm{Ct}$ value variation for six pairs of primers among all the samples. The variability of $\mathrm{Ct}$ values was highest for $A v E F 1 \alpha-1$, and the other genes showed slightly lower levels of variation (Fig. 2). However, none of the tested primer pairs exhibited a constant expression level among the samples. To determine their stability under specific experimental conditions, the 18 samples were further divided into two subsets, of which one was eight different tissues and the other was 10 samples with different levels of salinity stress.

In the different-tissues subset, the $\mathrm{Ct}$ values of the six primer pairs used varied from 16.2 to 26.2 cycles, which was similar to the range reported earlier among all samples. The highest and lowest average mean $\mathrm{Ct}$ values were detected in $A v G A P D H-2$ and $A v U B Q-2$, with 23.2 and 17.4, respectively. As expected, the $\mathrm{Ct}$ value variation among the six pairs of primers in the different-tissues group was the same as the total samples, ranging from 3.8 to 5.1. However, the range of $\mathrm{Ct}$ values of the six primer pairs in the salinity-stressed samples ranged from 16.4 to 24.8 , showing minor variation compared with the total samples and the different-tissues group. Not surprisingly, the variation of $\mathrm{Ct}$ values among each of the six primer pairs showed a narrow range, from 1.3 to 2.4 in salinity-stressed samples. Unlike the other candidate reference primer pairs, the distribution of $\mathrm{Ct}$ values of $A v E F 1 \alpha-1$ in the salinity-stressed samples and different-tissue samples showed obvious differences (Fig. 2). Given the various factors that can affect the accuracy of the results, such as variations in the amount of starting

Table 2. Ranking of candidate reference gene primer pairs of sword-leaf dogbane and the expression stability values analyzed by NormFinder (Andersen et al., 2004).

\begin{tabular}{|c|c|c|c|c|c|c|}
\hline \multirow[b]{2}{*}{ Ranking order } & \multicolumn{2}{|c|}{ Total samples $^{\mathrm{z}}$} & \multicolumn{2}{|c|}{ Different tissues $^{\mathrm{y}}$} & \multicolumn{2}{|c|}{ Salinity stress ${ }^{\mathrm{x}}$} \\
\hline & Primer pair & Stability value & Primer pair & Stability value & Primer pair & $\overline{\text { Stability value }}$ \\
\hline 1 & $A v A C T I N-2$ & 0.137 & $A v A C T I N-2$ & 0.327 & $A v G A P D H-2$ & 0.045 \\
\hline 2 & $A v G A P D H-2$ & 0.142 & $A v A C T I N-3$ & 0.369 & $A v b T U B-2$ & 0.073 \\
\hline 4 & $A v A C T I N-3$ & 0.292 & $A v b T U B-2$ & 0.512 & $A v U B Q-2$ & 0.120 \\
\hline 5 & $A v U B Q-2$ & 0.409 & $A v E F 1 \alpha-1$ & 0.603 & $A v A C T I N-3$ & 0.129 \\
\hline 6 & $A v E F 1 \alpha-1$ & 0.710 & $A v U B Q-2$ & 0.695 & $A v E F 1 \alpha-1$ & 0.258 \\
\hline
\end{tabular}

${ }^{\mathrm{z}}$ Combination of different tissues and salinity stress samples.

${ }^{\mathrm{y}}$ Young leaves, stems, and roots that dissected from seedlings, whole seedlings plants, germinated seeds, and senescent leaves, flowers, and perennial root that obtained from adult plants.

${ }^{\mathrm{x}}$ Salinity stress samples were consisted of mature leaves of sword-leaf dogbane adult plants that treated by $0,200,400,600$, and $800 \mathrm{~mm} \mathrm{NaCl}$ for 3 and $7 \mathrm{~d}$.

Table 3. Expression stability values of the candidate reference gene primer pairs of sword-leaf dogbane calculated using BestKeeper (Pfaffl et al., 2004).

\begin{tabular}{|c|c|c|c|c|c|c|c|c|c|}
\hline \multirow[b]{2}{*}{ Ranking order } & \multicolumn{3}{|c|}{ Total samples ${ }^{z}$} & \multicolumn{3}{|c|}{ Different tissues $^{\mathrm{y}}$} & \multicolumn{3}{|c|}{ Salinity stress ${ }^{\mathrm{x}}$} \\
\hline & Primer pair & $r$ & SD & Primer pair & $r$ & SD & Primer pair & $r$ & SD \\
\hline 1 & $A v A C T I N-2$ & 0.941 & 0.77 & $A v A C T I N-3$ & 0.966 & 1.16 & $A v b T U B-2$ & 0.992 & $\overline{0.35}$ \\
\hline 2 & $A v G A P D H-2$ & 0.931 & 0.82 & $A v A C T I N-2$ & 0.941 & 1.20 & $A v G A P D H-2$ & 0.99 & 0.53 \\
\hline 4 & $A v b T U B-2$ & 0.913 & 0.83 & $A v b T U B-2$ & 0.916 & 1.20 & $A v A C T I N-2$ & 0.968 & 0.50 \\
\hline 5 & $A v U B Q-2$ & 0.791 & 0.75 & $A v U B Q-2$ & 0.819 & 1.20 & $A v U B Q-2$ & 0.959 & 0.41 \\
\hline 6 & $A v E F 1 \alpha-1$ & 0.773 & 1.37 & $A v E F 1 \alpha-1$ & 0.864 & 1.03 & $A v E F 1 \alpha-1$ & 0.954 & 0.58 \\
\hline
\end{tabular}

${ }^{\mathrm{z}}$ Combination of different tissues and salinity stress samples.

${ }^{\mathrm{y}}$ Young leaves, stems, and roots that dissected from seedlings, whole seedlings plants, germinated seeds, and senescent leaves, flowers, and perennial root that obtained from adult plants.

${ }^{\mathrm{x}}$ Salinity stress samples were consisted of mature leaves of adult plants that treated by $0,200,400,600$, and $800 \mathrm{~mm} \mathrm{NaCl}$ for 3 and $7 \mathrm{~d}$. 

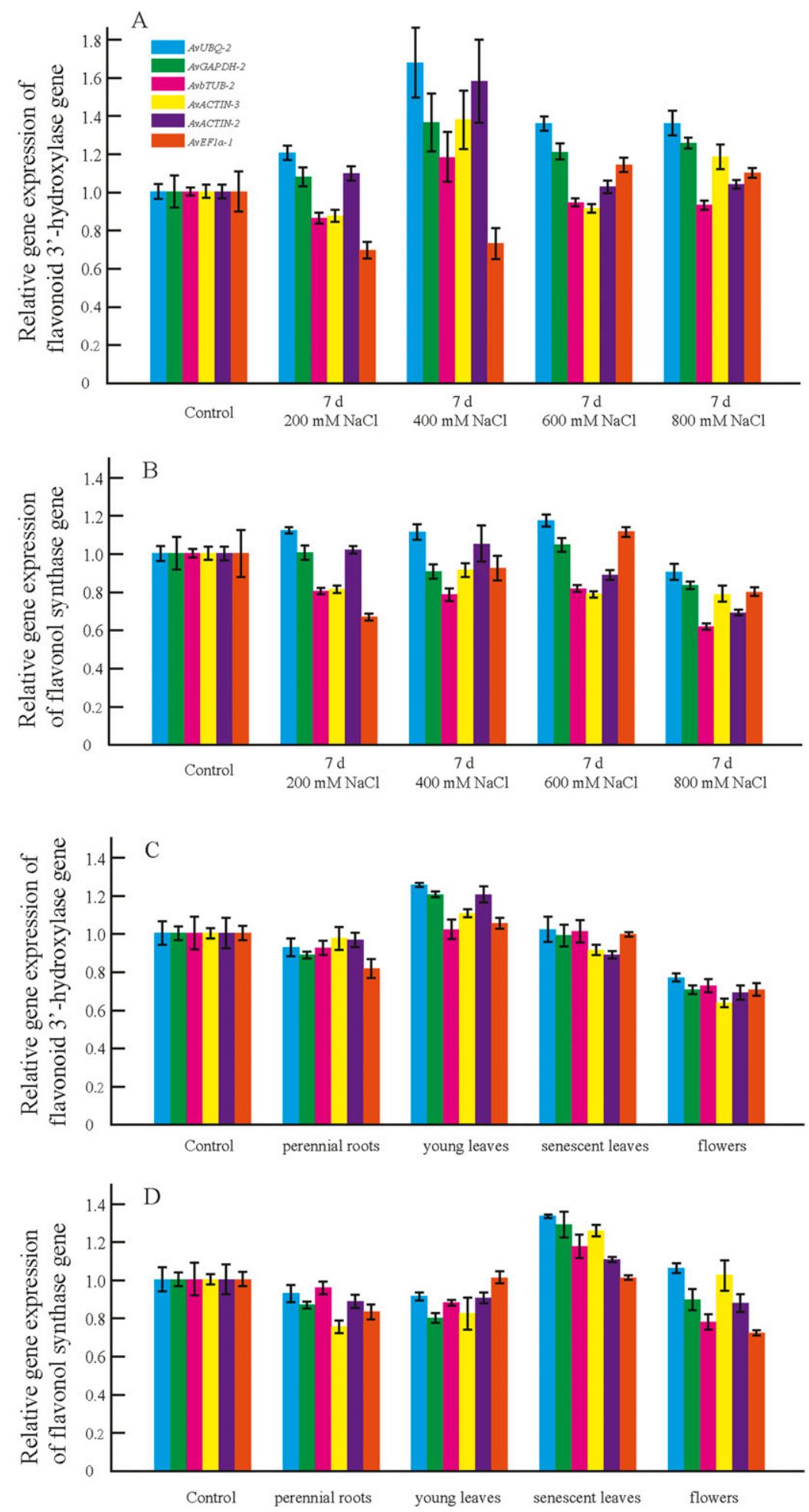

Fig. 4. Relative expression levels of two hyperoside biosynthesis terminal enzyme genes flavonoid $3^{\prime}$-hydroxylase $\left(F 3^{\prime} H\right)$ and flavonol synthase $(F L S)$ of swordleaf dogbane normalized by all candidate reference genes. (A) The relative expression level of $F 3^{\prime} H$ in the mature leaves of sword-leaf dogbane adult plant treated by $200,400,600$, and $800 \mathrm{~mm} \mathrm{NaCl}$ for $7 \mathrm{~d}$ normalized by different candidate reference genes $(A v U B O-2, A v G A P D H-2, A v b T U B-2, A v A C T I N-3, A v A C T I N-2$, and $A v E F 1 \alpha-1$ ); mature leaves of untreated sword-leaf dogbane adult plant were used as control. (B) The relative expression level of $F L S$ in the mature leaves of sword-leaf dogbane adult plant treated by $200,400,600$, and $800 \mathrm{~mm} \mathrm{NaCl}$ for $7 \mathrm{~d}$ normalized by different candidate reference genes $(A v U B Q-2, A v G A P D H-2$, $A v b T U B-2, A v A C T I N-3, A v A C T I N-2$, and $A v E F 1 \alpha-1)$; mature leaves of untreated sword-leaf dogbane adult plant were used as control. (C) The relative expression level of $F 3^{\prime} H$ in different tissues (perennial roots, young leaves, senescent leaves, and flowers) of sword-leaf dogbane adult plant treated by $200 \mathrm{~mm}$ $\mathrm{NaCl}$ for $7 \mathrm{~d}$ normalized by different candidate reference genes ( $A v U B Q-2, A v G A P D H-2, A v b T U B-2, A v A C T I N-3, A v A C T I N-2$, and $A v E F 1 \alpha-1)$; mature leaves of untreated sword-leaf dogbane adult plant were used as control. (D) The relative expression level of $F L S$ in different tissues (perennial roots, young leaves, senescent leaves, and flowers) of sword-leaf dogbane adult plant treated by $200 \mathrm{~mm} \mathrm{NaCl}$ for $7 \mathrm{~d}$ normalized by different candidate reference genes $(A v U B Q-2$, $A v G A P D H-2, A v b T U B-2, A v A C T I N-3, A v A C T I N-2$, and $A v E F 1 \alpha-1)$; mature leaves of untreated sword-leaf dogbane adult plant were used as control. Error bars show the $\mathrm{SE}$ calculated from three biological replicates. 
material between the samples, different inverse-transcription total RNA content, and subsequent operations of qRT-PCR, a direct comparison of the raw $\mathrm{Ct}$ values did not result in an accurate estimate of the expression stability of each reference gene. Therefore, the stability of the six primer pairs was estimated by more powerful tools.

GeNorm, NormFinder, and BestKeeper anAlyses. The average of the expression stability measures of the primer pairs (M) was calculated by the geNorm tool. When all 18 samples were considered, $A v A C T I N-3$ and $A v b T U B-2$ showed the lowest average expression stability value $(\mathrm{M}=0.414)$, and $A v E F 1 \alpha-1$ showed the highest $\mathrm{M}$ value of 0.875 (Fig. 3A). These results suggested that $A v A C T I N-3$ and $A v b T U B-2$ had the most stable expression, and $A v E F 1 \alpha-1$ had the highest level of expression variation. Among the eight samples in the differenttissues group, $A v A C T I N-2$ and $A v A C T I N-3$ showed the lowest average expression stability value $(\mathrm{M}=0.337)$, whereas $A v U B Q-2$ showed the highest $\mathrm{M}$ value of 0.988 (Fig. 3B). In the salinity-stressed samples group, $A v U B Q-2$ and $A v G A P D H-2$ showed the lowest average expression stability value $(\mathrm{M}=$ 0.129 ), whereas $A v E F 1 \alpha-1$ showed the highest M value of 0.258 (Fig. 3C). However, the level of variation we observed was not sufficient for an $M$ value greater than 1.5 in any of the experimental subsets, indicating that all of the primer sets met a reasonable standard for reference genes.

In addition, the geNorm tool was also used to determine the optimal number of reference genes required for accurate normalization among different experimental subsets. Among the 18 samples, these analyses showed that the pairwise variation $\mathrm{V}_{2 / 3}$ was higher than $0.15(\mathrm{~V}=0.151)$, whereas $\mathrm{V}_{3 / 4}$ was 0.117 , which indicated that three genes were required for reliable normalization of target genes in these samples (Fig. 3D). When the salinity-stressed samples were tested, all the pairwise variations dropped below the given threshold $(0.15$ Fig. 3D), indicating that one reference gene was adequate for normalization. However, all the pairwise variation values were more than 0.15 in the different-tissues group, indicating the large variation among these samples.

Subsequently, NormFinder was used to estimate the character of candidate reference primer pairs. On the basis of the results, $A v A C T I N-2$ (0.137) and $A v G A P D H-2$ (0.142) were identified as the two most stable primer pairs among the 18 samples (Table 2). Interestingly, $A v A C T I N-2$ (0.327) and $A v G A P D H-2$ (0.045) were also evaluated as the most stable primer pairs in the different-tissues group, and the salinitystressed group, respectively (Table 2). This was consistent with the results obtained with geNorm tool (Fig. 3B and $\mathrm{C}$ ). Although $A v E F 1 \alpha-1$ and $A v U B Q-2$ were the two most unstable primer pairs in the three experimental subsets, the largest stability value among them was 0.71 ( $A v E F 1 \alpha-1$, total samples) (Table 2), which was suitable for use as a reference gene according to the parameters of NormFinder.

Finally, the BestKeeper analysis revealed that the best correlations among the 18 samples were obtained for $A v A C T I N-2(r=$ $0.941)$, followed by $A v A C T I N-3(r=0.928)$ and $A v G A P D H-2(r=$ 0.931 ) with a probability value of 0.001 (Table 3 ). When only the salinity-stressed samples were considered, all the six primer pairs had a strong correlation according to the BestKeeper index $[r>0.95$ (Table 3)]. The top three BestKeeper scores in the different-tissues group were $A v A C T I N-3, A v A C$ $T I N-2$, and $A v G A P D H-2$. However, their standard deviation values were above the threshold (Table 3 ). In summary, the top two stable reference primer pairs based on the three assessment tools were $A v A C T I N-2 / A v G A P D H-2, A v A C T I N-2 /$ $A v A C T I N-3$, and $A v G A P D H-2 / A v b T U B-2$ in the total samples set, the different-tissues group, and the salinity-stressed set, respectively.

VALIDATION OF REFERENCE GENES. To evaluate the effectiveness of reference genes selected in this study, and to further validate their reliability, we examined the relative expression levels of two functional genes, $F 3^{\prime} H$ and $F L S$, which encode two hyperoside biosynthesis terminal enzymes. These were assessed using all six candidate reference primer pairs in the salinity-stressed group. After normalizing by all of the six candidate reference primer pairs, we found that $F 3^{\prime} H$ was expressed at a significantly lower level in samples treated with 200 and $400 \mathrm{~mm} \mathrm{NaCl}$ following normalization with $A v E F 1 \alpha-1$ compared with the other candidate reference primer pairs (Fig. 4A). The highest expression in $400 \mathrm{~mm}$ salinity samples was clearly shown by $F 3^{\prime} H$. The expression diversity of $F 3^{\prime} H$ normalized by $A v U B Q-2, A v G A P D H-2, A v b T U B-2, A v A C T I N-$ 3, and $A v A C T I N-2$ was fairly consistent among the salinitystressed sample set (Fig. 4A). Expression of FLS was relatively more stable when normalized by all six primer pairs (Fig. 4B), supporting the reliability of the selected candidate reference primer pairs. In contrast, the relative expression of $F 3^{\prime} H$ and $F L S$ in the different tissues treated by $200 \mathrm{~mm} \mathrm{NaCl}$ for $7 \mathrm{~d}$ was also normalized by these six candidate reference primer pairs. The qRT-PCR results showed slight differences in expression levels in each tissue, normalized by different candidate reference primer pairs (Fig. 4C and D).

\section{Discussion}

Although sword-leaf dogbane is an important herb in traditional Chinese medicine (TCM), its examination at a molecular level is lacking. However, this is likely to change with the development of biosynthesis, and the increasing interest in molecular investigation of TCM. The study of candidate reference genes for this species therefore has prospective significance. In this study, we successfully cloned partial sequences of five sword-leaf dogbane candidate reference genes, including $A v G A P D H, A v b T U B, A v U B Q, A v E F 1 \alpha$, and $A v A C T I N$ (Supplemental Table 2). On the basis of their sequences, we designed 12 pairs of primers that we used for qRT-PCR (Table 1). We evaluated six of these, covering all five candidate reference genes, under different experimental conditions.

Selection of reference genes is relevant given that qRT-PCR is the most widely used method to evaluate the expression levels of genes of interest (Gachon et al., 2004). Although it is common to use only one reference primer pair per gene (Garg et al., 2010; Jain et al., 2006; Liu et al., 2014), our results support the concept of using more than one (Table 1). However, the PCR efficiency of about half of these primer pairs was below $90 \%$, which has been considered a lower threshold for acceptability (Pfaffl et al., 2004). We found no correlations between PCR efficiency and primer location. Interestingly, two primer pairs of a candidate reference gene exhibited different PCR efficiencies (Table 1), suggesting that one primer pair is not enough for a comprehensive and rigorous estimation of the real expression level of genes. Careful selection of internal reference genes and evaluation of PCR efficiency are necessary to optimize evaluation of the expression levels of genes of interest. 
We used raw $\mathrm{Ct}$ values to make initial estimates of the amplification of candidate reference primer pairs for which PCR efficiency was greater than $90 \%$. However, the $\mathrm{Ct}$ value variation of six pairs of primers in our different-tissue sample group was larger than that in the salinity-stressed group (Fig. 2). We then further characterized these candidate reference primer pairs using GeNorm, NormFinder, and BestKeeper software, which are the three most widely used programs for evaluating candidate reference primer pairs. The results confirmed that the variation levels of six pairs of primers in the different-tissues group were larger than in the salinity-stressed group (Tables 2 and 3; Fig. 3). All of our results suggested the presence of some spatio-temporal variation in the expression of our selected housekeeping genes. They showed relatively stable expression under stresses such as salinity, although this is not surprising because sword-leaf dogbane is a halophyte, which is suitable for growth in a high-salinity environment.

In general, the expression results of targeted genes normalized by ideal reference genes are essentially consistent. In the present study, the $M$ value of six pairs of primers was less than 1.5 under all experimental conditions in this study (Fig. $3 \mathrm{~A}-\mathrm{C}$ ), suggesting that all the six primer pairs could be used as reference genes to normalize expression results. However, the variation of $M$ value in salinity-stressed samples was much smaller than that in the different-tissues group. Consistent with this result, the $\mathrm{V}_{\mathrm{n}} / \mathrm{V}_{\mathrm{n}+1}$ value detected in the salinity-stressed group was also smaller than that in the different-tissues group (Fig. 3D), implying slight expression changes of the five reference genes in the salinity-stressed group, which is consistent with the distribution of $\mathrm{Ct}$ values (Fig. 2). A similar conclusion was obtained from the parameters validated by NormFinder and BestKeeper (Tables 2 and 3). All our results support the conclusion drawn by previous studies that no reference gene is universal for all experiments (Gutierrez et al., 2008a; Hruz et al., 2011; Liu et al., 2014; Maroufi et al., 2010). Specifically, in our research, the most suitable reference primer pairs in high salinity were $A v G A P D H-2$ and $A v b T U B-2$, and the most suitable reference primer pairs across different tissues were $A v A C T I N-2$ and $A v A C T I N-3$.

Hyperoside is one of the most effective medicinal substances found in sword-leaf dogbane (Yamatsu et al., 2015). The genes $F 3^{\prime} H$ and $F L S$, encode synthetases involved in the final several steps of hyperoside biosynthesis. Briefly, the expression profiles of these two target genes obtained using the six reference primer pairs were affected similarly by varying the salinity level and the tissue (Fig. 4), further supporting the applicability of our identified reference genes. Overall, our results support the importance of validating reference genes and gene-specific primer pairs to be used in normalizing the relative expression of genes of interest.

\section{Literature Cited}

Aithal, M.G.S. and N. Rajeswari. 2015. Validation of housekeeping genes for gene expression analysis in glioblastoma using quantitative real-time polymerase chain reaction. Brain Tumor Res. Treat. 3:2429.

Andersen, C.L., J.L. Jensen, and T.F. Ørntoft. 2004. Normalization of real-time quantitative reverse transcription-PCR data: A modelbased variance estimation approach to identify genes suited for normalization, applied to bladder and colon cancer data sets. Cancer Res. 64:5245-5250.

Artico, S., S.M. Nardeli, O. Brilhante, M.F. Grossi-de-Sa, and M. Alves-Ferreira. 2010. Identification and evaluation of new reference genes in Gossypium hirsutum for accurate normalization of real-time quantitative RT-PCR data. BMC Plant Biol. 10:49.

Bustin, S.A. and S. Dorudi. 1998. Molecular assessment of tumour stage and disease recurrence using PCR-based assays. Mol. Med. 4:389-396.

Bustin, S.A. and R. Mueller. 2005. Real-time reverse transcription PCR (qRT-PCR) and its potential use in clinical diagnosis. Clin. Sci. (Lond.) 109:365-379.

Dean, J.D., P.H. Goodwin, and T. Hsiang. 2002. Comparison of relative RT-PCR and northern blot analyses to measure expression of $\beta$-1,3-glucanase in Nicotiana benthamiana infected with Colltotrichum destructivum. Plant Mol. Biol. Rpt. 20:347-356.

Gachon, C., A.C. Mingam, and B.N.D. Charrier. 2004. Real-time PCR: What relevance to plant studies? J. Expt. Bot. 55:1445-1454.

Garg, R., A. Sahoo, A.K. Tyagi, and M. Jain. 2010. Validation of internal control genes for quantitative gene expression studies in chickpea (Cicer arietinum L.). Biochem. Biophys. Res. Commun. 396:283-288.

Gutierrez, L., M. Mauriat, S. Guenin, J. Pelloux, J.F. Lefebvre, R. Louvet, C. Rusterucci, T. Moritz, F. Guerineau, C. Bellini, and O. Van Wuytswinkel. 2008a. The lack of a systematic validation of reference genes: A serious pitfall undervalued in reverse transcriptionpolymerase chain reaction (RT-PCR) analysis in plants. Plant Biotechnol. J. 6:609-618.

Gutierrez, L., M. Mauriat, J. Pelloux, C. Bellini, and O.V. Wuytswinkel. 2008b. Towards a systematic validation of references in real-time RTPCR. Plant Cell 20:1734-1735.

Hong, S.Y., P.J. Seo, M.S. Yang, F. Xiang, and C.M. Park. 2008. Exploring valid reference genes for gene expression studies in Brachypodium distachyon by real-time PCR. BMC Plant Biol. 8:112. Hruz, T., M. Wyss, M. Docquier, M.W. Pfaffl, S. Masanetz, L. Borghi, P. Verbrugghe, L. Kalaydjieva, S. Bleuler, O. Laule, P. Descombes, W. Gruissem, and P. Zimmermann. 2011. RefGenes: Identification of reliable and condition specific reference genes for RT-qPCR data normalization. BMC Genomics 12:156.

Huggett, J., K. Dheda, S. Bustin, and A. Zumla. 2005. Real-time RTPCR normalisation; strategies and considerations. Genes Immun. 6:279-284.

Jain, M., A. Nijhawan, A.K. Tyagi, and J.P. Khurana. 2006. Validation of housekeeping genes as internal control for studying gene expression in rice by quantitative real-time PCR. Biochem. Biophys. Res. Commun. 345:646-651.

Kibbe, W.A. 2007. OligoCalc: An online oligonucleotide properties calculator. Nucleic Acids Res. 35:W43-W46.

Kim, D.W., T. Yokozawa, M. Hattori, S. Kadota, and T. Namba. 2000. Effects of aqueous extracts of Apocynum venetum leaves on spontaneously hypertensive, renal hypertensive and $\mathrm{NaCl}$-fed-hypertensive rats. J. Ethnopharmacol. 72:53-59.

Kong, Q., J. Yuan, L. Gao, S. Zhao, W. Jiang, Y. Huang, and Z. Bie. 2014. Identification of suitable reference genes for gene expression normalization in qRT-PCR analysis in watermelon. PLoS One 9:e90612.

Liu, M., J. Jiang, X. Han, G. Qiao, and R. Zhuo. 2014. Validation of reference genes aiming accurate normalization of qRT-PCR data in Dendrocalamus latiflorus Munro. PLoS One 9:e87417.

Lv, L., D. Zhang, B. Sun, Y. Hu, M. Yan, K. Liu, X. Li, and L. Ren. 2016. Apocynum leaf extract inhibits the progress of atherosclerosis in rats via the AMPK/mTOR pathway. Pharmazie 72:41-48.

Maroufi, A., E.V. Bockstaele, and M.D. Loose. 2010. Validation of reference genes for gene expression analysis in chicory (Cichorium intybus) using quantitative real-time PCR. BMC Mol. Biol. 11:15.

Nolan, T., R.E. Hands, and S.A. Bustin. 2006. Quantification of mRNA using real-time RT-PCR. Nat. Protoc. 1:1559-1582.

Pfaffl, M.W., A. Tichopad, C. Prgomet, and T.P. Neuvians. 2004. Determination of stable housekeeping genes, differentially regulated target genes and sample integrity: BestKeeper-Excel based tool using pair-wise correlations. Biotechnol. Lett. 26:509-515. 
Radoni, A., S. Thulke, I.M. Mackay, O. Landt, W. Siegert, and A. Nitsche. 2003. Guideline to reference gene selection for quantitative real-time PCR. Biochem. Biophys. Res. Commun. 313:856-862.

Sinha, P., V.K. Singh, V. Suryanarayana, L. Krishnamurthy, R.K. Saxena, and R.K. Varshney. 2015. Evaluation and validation of housekeeping genes as reference for gene expression studies in pigeonpea (Cajanus cajan) under drought stress conditions. PLoS One 10:e0122847.

Sturzenbaum, S.R. and P. Kille. 2001. Control genes in quantitative molecular biological techniques: The variability of invariance. Comp. Biochem. Physiol. 130:281-289.

Vandesompele, J., K.D. Preter, F. Pattyn, B. Poppe, N.V. Roy, A.D. Paepe, and F. Speleman. 2002. Accurate normalization of real-time quantitative RT-PCR data by geometric averaging of multiple internal control genes. Genome Biol. 3:0034.I-0034.II.

Wang, M., Z. Xu, A. Ding, and Y. Kong. 2018. Genome-wide identification and expression profiling analysis of the xyloglucan endotransglucosylase/hydrolase gene family in tobacco (Nicotiana tabacum L.). Genes (Basel) 9:273.
Wang, W., X. Liang, D. Fu, R. Tie, W. Xing, L. Ji, F. Liu, H. Zhang, and R. Li. 2015. Apocynum venetum leaf attenuates myocardial ischemia/reperfusion injury by inhibiting oxidative stress. Amer. J. Chin. Med. 43:71-85.

Wong, M.L. and J.F. Medrano. 2005. Real-time PCR for mRNA quantitation. Biotechniques 39:75-85.

Xie, W., X. Zhang, T. Wang, and J. Hu. 2012. Botany, traditional uses, phytochemistry and pharmacology of Apocynum venetum L. (Luobuma): A review. J. Ethnopharmacol. 141:1-8.

$\mathrm{Xu}, \mathrm{Z}$., J. Zhou, C. Zhang, and Y. Li. 2018. Review of current research and utilization status of Apocynum venetum germplasm in China. Chinese Bul. Bot. 53:382-390.

Yamatsu, A., Y. Yamashita, I. Maru, J. Yang, J. Tatsuzaki, and M. Kim. 2015. The improvement of sleep by oral intake of GABA and Apocynum venetum Leat extract. J. Nutr. Sci. Vitaminol. (Tokyo). 61:182-187.

Zhang, G., X. Qian, and G. Gu. 2005. Apocynum venetum L. resources in Shanxi, Hubei, Henan and Shandong provinces and its cultivation techniques. Chinese Wild Plant Resources. 24:26-27, 50. 
Supplemental Table 1. Primers used to amplify the homologous reference genes of sword-leaf dogbane.

\begin{tabular}{ll}
\hline Primer name & \multicolumn{1}{c}{ Primer sequence $\left(5^{\prime} \rightarrow 3^{\prime}\right)$} \\
\hline AvACTIN(H)-F & ATGAYATGGAGAARATWTGGCATC \\
AvACTIN(H)-R & ARCAAYTGGGATGAYATGGAGAA \\
AvGAPDH(H)-F & GGTGGTKCATGAGGARTTTGG \\
AvGAPDH(H)-R & GCMAAGTACATGGCTTACATGTT \\
AvbTUB(H)-F & AGAAYTGTGAYTGYCTYCAAGG \\
AvbTUB $(\mathrm{H})-\mathrm{R}$ & CTCCTGAACATAGCWGTRAAYT \\
AvUBQ $(\mathrm{H})-\mathrm{F}$ & AYATYCAGAAGGAGTCYACCCT \\
AvUBQ $(\mathrm{H})-\mathrm{R}$ & GTTGATCTTYGCYGGMAAGCA \\
AvEF1 $\alpha(\mathrm{H})-\mathrm{F}$ & GATTGAGAGGTCYACCAACCTYGA \\
AvEF1 $\alpha(\mathrm{H})-\mathrm{R}$ & CATCTTAACCATACCAGCATCA \\
\hline
\end{tabular}


Supplemental Table 2. Partial coding sequences (CDS) of sword-leaf dogbane candidate reference genes.

CGTGTTGCTCCTGAGGAGCACCCTGTGCTGCTTACTGAGGCGCCTCTTAATCCCAAGGCTAATAGAGAGAAGATGACTCAAATTATGTTTGAG ACCTTTAATGTACCTGCAATGTATGTTGCCATTCAGGCTGTTCTTTCTCTATATGCTAGTGGTCGTACAACTGGTATTGTACTGGATTCTGGTGA TGGTGTGAGTCATACTGTGCCAATCTATGAAGGGTATGCTCTACCCCATGCAATTCTTCGTCTTGATCTTGCAGGTCGTGACCTCACAGATCA ACTCATGAAGATCCTCACTGAAAGAGGTTACATGTTCACCACCTCTGCTGAACGGGAAATTGTCCGTGATATCAAAGAAAAGTTAGCATATGTG GCTCTTGACTATGAGCAGGAGCTTGAAACGGCAAAAAGCAGCTCTTCTCTTGAGAAGAACTATGAATTGCCTGATGGACAGGTCATTACAATTG GAAATGAGAGATTCCGTTGCCCAGAGGTCCTATTCCAGCCTTCAATGATCGGTATGGAAGCTGCTGGTATCCATGAGACTACCTACAATTCCAT CATGAAGTGTGATGTGGATATCAGGAAGGATCTCTATGGTAACATTGTGCTCAGTGGTGGTTCAACTATGTTCCCTGGTATTGCTGACCGTATG AGCAAGGAAATCACTGCATTGGCTCCCAGCAGCATGAAGATTAAGGTGGTTGCACCACCAGAGAGAAAGTACAGTGTCTGGATTGGAGGATCA ATTCTTGCATCACTCAGTACCTTCCAGCAGATGTGGATCTCGAAGGCTGAGTATGAAGAGTCTGGCCCATCAATTGTCCACAGGAAGTGCTTC TAAGATGCTACTCTTGCTTGAGATGGTGAAATTTTTTCTCCACCCGTCTAGTTGATCAATCTTTCATATTATGTTACAAGTGGTGTCAGTTTG CCGATATGATGGAAGTTTTGAAGGTGGGGTTCTAAAGGAGGGCATGGTGT

>sword-leaf dogbane GAPDH CDS sequence

GGAGTCTTCATGGCACCCTCCGAGTTTTAGATGAATCTACCTTGGAAATCAATGGGAAACAAATAAAAATCACCAGCAAAAGGGATCCAGCTCA AATTCCATGGGGTGATTATGGGGCTGACTATGTTGTTGAATCATCTGGAGTTTTCACTACAGTTGAGAAGGCNTCAGCACACAAGAAGGGTGGT GCCAAAAAGGTTGTAATCTCTGCTCCATCTGCTGATGCACCTATGTTCGTGGTGGGAGTAAATGAGTTTACCTACAAACCAAGCATGGATGTTG TTTCCAATGCTAGCTGTACTACTAACTGCCTTGCTCCTCTTGCCAAGGTGGTCCATGAGGAGTTTGGCATTCTAGAGGGCTTAATGACAACTGTC CATGCAACCACAGCTACACAAAAGACTGTTGATGGTCCTTCAATGAAGGATTGGCGTGGAGGCCGTGGAGCAGGACAAAATATTATCCCTAGT TCTACTGGTGCAGCAAAGGCTGTTGGAAAGGTTCTTCCAGAACTAAATGGCAAACTTACTGGAATGGCCTTCCGTGTTCCAACACCTAATGTCT CTGTGGTGGACTTAACTTGTCGACTTGAGAAGAGTGCTTCTTACGAAGATGTCAAGGCAGCCATCAAGTATGCATCAGAGGGTCCTCTAAAGGG CATTCTTGGATACACTGATGACGATGTCGTCTCCAATGATTTTATTGGTGATTCAAGGTCAAGCATATTTGATGCGAAGGCAGGCATTGGCCTGA GTGATTCTTTCATGAAATTTGTTTCGTGGTATGACAACGAGTGGGGTTACAGCAACCGAGTTTTGGATCTGATAGAGCACATGGCACTGGTAGCA GCCACTCGCTAAAACTTTGAGAGGCACTTCCAGATGCAATTTTCGACCTAGAAAAGAGTTCAGTTATGAGCATGGCTACAAATTTGTTAGGAAC TTAGTTATATACGTTTTATGTTTGGCTGGGCTTTAGCGTTTGTGCTGTAGTTGCCATAGTTGCTCCAATGTGTCATTGGGAATAATGAATTAAG GAATGTACAAGCTCTTCGAATAAAGAGTGGATTATGGATACACTTGTGTTTTGCACAATTATATCCCCGCCACTTTTTACCCGACGCACAGCG GCGGTGGAGGGGGGGGAGAAAAGGA

>sword-leaf dogbane $b$-TUB CDS sequence

GAGAAAGGAAGCTGAGAATTGTGACTGCCTTCAAGGTTTTCAAGTTTGCCACTCTCTTGGTGGTGGTACAGGTTCTGGTATGGGTACCTTGTTGA TTTCAAAAATCAGGGAGGAATACCCAGACAGAATGATGCTCACATTTTCTGTGTTCCCATCTCCAAAGGTGTCCGATACTGTGGTTGAGCCATAC AATGCCACCCTTTCAGTGCATCAACTTGTGGAGAATGCAGATGAATGCATGGTGCTTGACAATGAAGCTTTATATGATATCTGCTTCAGAACC CTTAAGCTTACTACACCTAGCTTTGGTGATTTGAATCACTTGATATCTGCTACGATGAGTGGGGTCACTTGCTGTCTCCGATTCCCCGGTCAGC TCAATTCTGATTTAAGGAAGCTTGCTGTCAACCTTATCCCATTTCCCCGTCTGCACTTCTTTATGGTTGGTTTTGCTCCTTTGACCTCACGTGGC TCTCAGCAGTATCGTGCACTTACAGTTCCTGAACTTACCCAACAAATGTGGGATGCAAAGAACATGATGTGTGCTGCTGATCCTCGACACGGG CGTTACCTCACTGCCTCAGCCATGTTCCGAGGGAAAATGAGTACCAAGGAAGTGGATGAACAGATGATTAATGTCCAAAACAAGAACTCTTC ATACTTTGTTGAATGGATCCCTAACAATGTCAAGTCCAGTGTCTGTGACATCCCACCCAGGGGGCTTTCAATGGCTTCCACCTTCATTGGTA ACTCAACATCCATCCAGGAGATGTTCAGGAGAGTGAGCGAGCAGTTTACTGCTATGTTCAGGAGGAAGGCTTTCTT

$>$ sword-leaf dogbane $U B Q$ CDS sequence

CGAATCCCTAGTGCCCTGGCGATACACATCCAGAAGGAGTCCACCCCTTCACTTGGTGCTTAGGCTCCGAGGTGGGATGCAAATATTTGTGAA GACCCTAACCGGAAAGACCATCACTCTGGAGGTCGAGAGCTCTGACACCATCGATAACGTGAAGGCTAAAATTCAGGACAAGGAGGGTATTCC CCCAGACCAACAGAGGCTGATCTTTGCTGGTAAGCAGCTGGAAGACGGGCGTACTCTTGCTGACTATAACATTCAGAAGGAGTCTACCCTTCA CTTGGTCCTCCGGCTTAGGGGAGGAATGCAGATTTTCGTGAAGACCTTGACCGGGAAGACCATAACTCTGGAGGTGGAGAGCTCTGATACCAT CGACAACGTGAAGGCCAAAATTCAGGACAAGGAGGGAATCCCACCTGACCAGCAGAGGTTGATCTTTGCTGGAAAGCGGCTGGAGTATGGTC TCACTCTTGCTGATTACATCTTTCTCAGGGAGTCCACCCTTCACTTGGTACTTAGGCTGAGAGGTGGGATGCAAATATTTGTGAAGACTTTGAC CGGAAAGACCATCACTCTGGAGGTTGATAGCTCAGACACCATTGATAACGTGAAGGCTAAAATCCAGGACAAGGAGGGTATTCCCCCTGACCA ACAGAGGCTGATCTTCGCTGGTAAGCAGCTGGAGGATGGCCGGACTCTGGCTGACTACAACATTCAGAAGGAGTCCACTCTCCACTTGGTTCT TCGTCTCAGTGGTGGCGTCTACATTGTAATCTGGATAGCGTATGGAAGCATGACTTGTATGTCTGTC

$>$ sword-leaf dogbane $E F 1 \alpha$ CDS sequence

CAATCTCTGGTTTTGAGGGAGACAACATGATTGAGAGGTCTACCAACCTTGACTGGTACAAGGGCCCAACCCTTCTTGAGGCTCTTGACCTGATT TCTGAGCCCAAGAGGCCCTCAGACAAGCCACTCCGGCTCCCACTTCAGGATGTCTACAAGATTGGTGGAATTGGTACTGTCCCTGTGGGTCGT GTTGAAACTGGTGTCCTCAAGCCTGGTATGGTTGTGACCTTTGGCCCAACTGGTCTGACAACTGAAGTTAAGTCAGTTGAGATGCACCATGAA GCGCTACAGGAGGCTCTTCCAGGTGACAATGTTGGGTTCAATGTGAAGAATGTTGCTGTTAAGGATCTCAAGCGTGGTTTTGTTGCATCCAAC TCCAAGGATGACCCAGCCAAGGGGGCTTCCAGCTTCACCTCGCAGGTTATCATCATGAACCACCCTGGCCAGATTGGAAATGGATATGCTCCA GTGCTTGACTGCCACACCTCTCACATTGCTGTCAAGTTTTCTGAGCTCTTGACCAAGATTGACAGACGATCTGGTAAGGAGCTCGAGAAGGAGC CCAAGTTTTTGAAGAATGGTGATGCTGGTATGGTTAAGATGATTCC 